\title{
Informing Pharmacokinetic Models With Physiological Data: Oral Population Modeling of L-Serine in Humans
}

\author{
J. R. Bosley ${ }^{1 *}$, Elias Björnson ${ }^{2,3}$, Cheng Zhang ${ }^{4}$, Hasan Turkez ${ }^{5}$, Jens Nielsen ${ }^{3}$, \\ Mathias Uhlen ${ }^{4}$, Jan Borén ${ }^{2}$ and Adil Mardinoglu ${ }^{4,6 *}$
}

${ }^{1}$ Clermont Bosley LLC, Philadelphia, PA, United States, ${ }^{2}$ Department of Molecular and Clinical Medicine, University of Gothenburg and Sahlgrenska University Hospital, Gothenburg, Sweden, ${ }^{3}$ Department of Biology and Biological Engineering, Chalmers University of Technology, Gothenburg, Sweden, ${ }^{4}$ Science for Life Laboratory, KTH-Royal Institute of Technology, Stockholm, Sweden, ${ }^{5}$ Department of Medical Biology, Faculty of Medicine, Atatürk University, Erzurum, Turkey, ${ }^{6}$ Centre for Host-Microbiome Interactions, Faculty of Dentistry, Oral and Craniofacial Sciences, King's College London, London, United Kingdom

\section{OPEN ACCESS}

Edited by:

George D Loizou,

Health and Safety Laboratory, United Kingdom

Reviewed by: José Augusto Guimarães Morais, Universidade de Lisboa, Portugal Amir Sadeghi,

University of Eastern Finland, Finland

*Correspondence:

J. R. Bosley

jbosley@clerbos.com Adil Mardinog/u adilm@scilifelab.se

Specialty section: This article was submitted to

Predictive Toxicology,

a section of the journal Frontiers in Pharmacology

Received: 25 December 2020 Accepted: 30 April 2021

Published: 13 May 2021

Citation:

Bosley J R, Björnson E, Zhang C, Turkez H, Nielsen J, Uhlen M, Borén J and Mardinoglu A (2021) Informing

Pharmacokinetic Models With Physiological Data: Oral Population Modeling of L-Serine in Humans.

Front. Pharmacol. 12:643179.

doi: 10.3389/fphar.2021.643179
To determine how to set optimal oral L-serine (serine) dose levels for a clinical trial, existing literature was surveyed. Data sufficient to set the dose was inadequate, and so an $(n=10)$ phase I-A calibration trial was performed, administering serine with and without other oral agents. We analyzed the trial and the literature data using pharmacokinetic (PK) modeling and statistical analysis. The therapeutic goal is to modulate specific serine-related metabolic pathways in the liver using the lowest possible dose which gives the desired effect since the upper bound was expected to be limited by toxicity. A standard PK approach, in which a common model structure was selected using a fit to data, yielded a model with a single central compartment corresponding to plasma, clearance from that compartment, and an endogenous source of serine. To improve conditioning, a parametric structure was changed to estimate ratios (bioavailability over volume, for example). Model fit quality was improved and the uncertainty in estimated parameters was reduced. Because of the particular interest in the fate of serine, the model was used to estimate whether serine is consumed in the gut, absorbed by the liver, or entered the blood in either a free state, or in a protein- or tissue-bound state that is not measured by our assay. The PK model structure was set up to represent relevant physiology, and this quantitative systems biology approach allowed a broader set of physiological data to be used to narrow parameter and prediction confidence intervals, and to better understand the biological meaning of the data. The model results allowed us to determine the optimal human dose for future trials, including a trial design component including IV and tracer studies. A key contribution is that we were able to use human physiological data from the literature to inform the PK model and to set reasonable bounds on parameters, and to improve model conditioning. Leveraging literature data produced a more predictive, useful model.

Keywords: Pharmacokinectics, L-Serine (ser), systems biology, NAFLD (non alcoholic fatty liver disease), oral supplementation 


\section{INTRODUCTION}

L-Serine (serine), a non-essential amino acid (AA), is de novo synthesized from glucose via 3-phosphoglycerate/3phosphoserine and via interconversion of glycine. It can be obtained from diet, the degradation of dietary proteins and phospholipids and consumed in the production of pyruvate, amino acids (glycine, L-cysteine), and sphingosine and phosphatidyl serine. It also may be racemized to $\mathrm{D}$-serine. Serine has attracted interest in the treatment of neurodegenerative diseases (de Koning et al., 2003), including Amyotrophic Lateral Sclerosis (Levine et al., 2017) and neuropathy (Garofalo et al., 2011; Scherer, 2011; Gantner et al., 2019).

As shown in Figure 1 (after (de Koning et al., 2003)), serine can be part of key metabolic pathways associated with proteins and carbohydrates, and supplemental serine is expected to affect hepatic metabolism. Mardinoglu et al have used Genome Scale Metabolic (GEM) modeling to infer that supplementation of serine may affect hepatic pathways that are relevant to the pathology of non-alcoholic fatty liver disease and steatohepatitis (NASH) (Mardinoglu et al., 2014). Experimentally, a positive effect of serine therapy had been reported for alcoholic fatty liver in mice and rats (Sim et al., 2015). This led to a clinical trial of oral serine, showing a significant reduction in hepatic fat (assessed via imaging) with concurrent reduction of liver tissue fibrotic serum markers (Mardinoglu et al., 2017). The trial data and further metabolic modeling analysis was used to generate a more comprehensive therapy approach, supplementation of metabolic cofactor formulation, of which serine is one of the component (described in Mardinoglu et al., 2017, 2019). Accurate modeling of serine, accounting for endogenous production and clearance, was needed to set the level of serine as part of this therapeutic cocktail.

Here, we aim to identify the optimal human dose of serine in this formulation to obtain the best therapeutic effect, and to avoid adverse effects in future clinical trials. Understanding clinical data and modeling the $\mathrm{PK}$ is complicated by the endogenous production of serine, predominantly (perhaps solely) from the kidney (Pitts and MacLeod, 1972; Kalhan and Hanson, 2012) leading to a measurable and significant baseline level in the blood.

\section{Review and Analysis of Literature Data}

PLASMA METABOLOMICS ANALYSIS Measurement of plasma levels of metabolites was performed using LC-MS. Briefly, the liquid chromatography-tandem mass spectrometry (LC-MS/MS) platform was based on a Waters ACQUITY ultraperformance liquid chromatography (UPLC) system and a Thermo-Finnigan LTQ mass spectrometer operated at nominal mass resolution, which was equipped with an electrospray ionization (ESI) source and a linear ion trap (LIT) mass analyzer. The sample extract was dried and then reconstituted in acidic or basic LC-compatible solvents, each of which contained 12 or more injection standards at fixed concentrations. One aliquot was analyzed using acidic positive

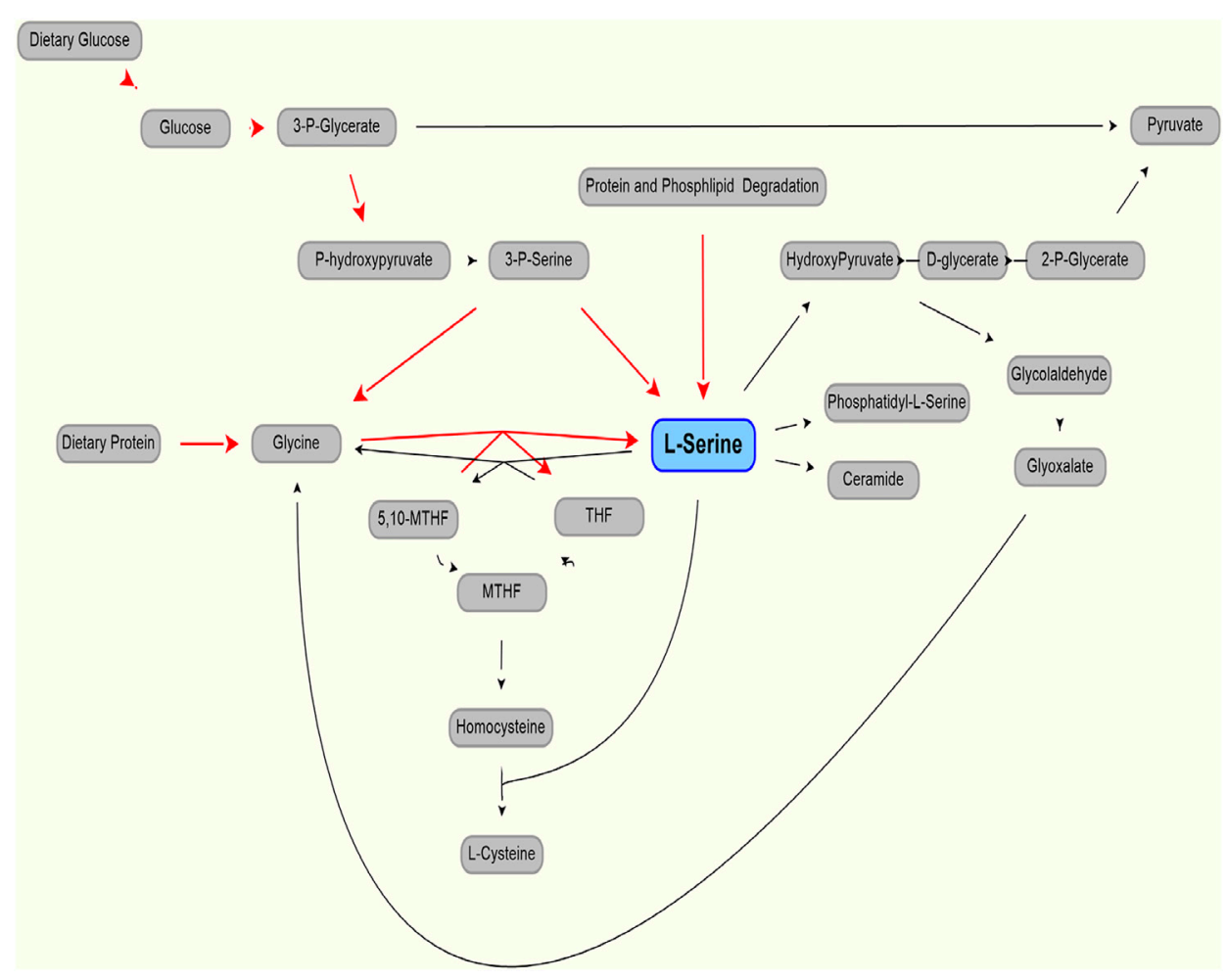

FIGURE 1 | Production (red line) and use (black lines) of L-serine in the body. Modified from (de Koning et al., 2003). 
ion-optimized conditions and the other was analyzed using basic negative ion-optimized conditions in two independent injections using separate dedicated columns (Waters UPLC BEH C18-2.1× $100 \mathrm{~mm}, 1.7 \mathrm{~lm}$ ). Extracts reconstituted in acidic conditions were gradient eluted using water and methanol containing $0.1 \%$ formic acid, whereas the basic extracts, which were also eluted using water/methanol, contained $6.5 \mathrm{mM}$ ammonium bicarbonate. The MS analysis alternated between MS and data-dependent MS/MS scans using dynamic exclusion, and the scan range was from 80 to $1,000 \mathrm{~m} / \mathrm{z}$.

\section{Published PK Analysis of Serine Is Sparse. Data From the Literature Are Given Below} VOLUME OF DISTRIBUTION (Pitts and MacLeod, 1972) reported values for volume of distribution of 199 and $360 \mathrm{~L}$ for dogs weighing 20 and $28 \mathrm{~kg}$ ( $10 \mathrm{~L} / \mathrm{kg}$ and $12.9 \mathrm{~L} / \mathrm{kg}$, respectively).

BASELINE CONCENTRATION A mean level (for five subjects) of 1.12 (with SEM of 0.046$) \mathrm{mg} / 100 \mathrm{ml}$, equivalent to 106 (4.3) umol/L was reported (Stein and Moore, 1954). These authors also cite a baseline serine level of $1.2 \mathrm{mg} / 100 \mathrm{ml}$, equivalent to $114 \mathrm{umol} / \mathrm{L}$, as being reported (Kurt Schreier and Plückthun, 1950). A baseline serine value of $0.97(0.07) \mathrm{mg} /$ $100 \mathrm{ml}$, or 92.3 (6.3) umol/L was reported (Frame, 1958). Neis et al. (Neis et al., 2017), reported a value, measured in the radial artery, of 97.2 (4.7) umol/L and a value from the renal vein of 130 (8.4) umol/L, a positive difference (that is, kidneys releasing serine) of $32.8 \mathrm{umol} / \mathrm{L}$. These data show the importance of rigorous methodology and complete reporting of data. Using a standard value for kidney blood perfusion of $1.2 \mathrm{~L} / \mathrm{m}$ (Calzia et al., 2005), and assuming that the assays capture all serine in the blood, we can estimate the net endogenous renal release of serine as approximately $4.2 \mathrm{mg} / \mathrm{min}$ or $252 \mathrm{mg} / \mathrm{h}$ from the kidney. A much higher estimate, which incorporates more detail and, estimates serine production to be $150 \mathrm{umol} / / \mathrm{kg} / \mathrm{h}$ (Kalhan and Hanson, 2012), or about 1,100 mg/h. Those authors suggest the possibility of an upregulation of serine production in the liver (but not in the kidney) during isocaloric protein restriction.

Wilcox et al. (Wilcox et al., 1985), show a mean baseline level of serine of approximately 131 (SD of $18.5 \mathrm{SD}$ ) $\mathrm{ug} / \mathrm{ml}$ for nonpsychotic control subjects. Interestingly, the baseline level shown for psychotics was 202.6 (SD 38) ug/ml, a difference that was reported as strongly significant $(p<0.0001)$. These researchers determined an approximately 50\% lower serine hydroxy methyl transferase (SHMT) enzyme activity in psychotic patients than in controls. The SHMT enzyme cleaves serine in the pathway synthesizing glycine from serine. Unfortunately, the order of magnitude for the baseline values reported is inconsistent with other reports $(131 \mathrm{ug} / \mathrm{mL}$ is about 1,247 umol/L, about 10 fold higher than other sources).

VARIABILITY DURING THE DAY Detailed analysis of other amino acids show a time variation in concentration after meals in pigs (Stoll et al., 1998), and a similar variation in human serine production might be expected. Stoll also pointed out that $40 \%$ of dietary serine was extracted by the liver in the first pass.

PK DATA/EXPERIMENTS Wilcox et al. (Wilcox et al., 1985), also administered an oral bolus of $4 \mathrm{mM} / \mathrm{kg}$ of serine to 12 actively psychotic patients, and 10 nonpsychotic subjects. This is equivalent to about a $29.5 \mathrm{~g}$ dose, for a $70 \mathrm{~kg}$ subject. Using the oral dosing data, they found a range for serine elimination halflife of between 1.85 and $14.81 \mathrm{~h}$ presumably due, at least in part, to SHMT level differences. There was a bimodal difference between active or previously active psychotic patients, and non-psychotic patients with no such history. The variability for psychotic patients was also much larger than for nonpsychotic subjects. The mean for non-psychotics was approximately $3.23 \mathrm{~h}$, with a standard error of the mean of $0.08 \mathrm{~h}$. The differences reported in SHMT may cause the longer half-life values and the higher baseline values. Clearly these factors must be considered in safe serine dosing in psychotic patients. Again the apparent discrepancy in units is noted.

PRODUCTION Endogenous production, at some level, clearly must be represented in the model. An accurate PK model must include physiological knowledge to some extent, as it must account for endogenous serine and accurate serine clearance. Indeed, because of the oral dose and the time-varying nature of serine concentration, the use of mechanistic understanding and literature data pertaining to parameters with physiological meaning were necessary to fit and validate our dosing model.

CLEARANCE MECHANISM The human kidney extracts AAs (significantly, glutamine and glycine) and secretes other amino acids (in largest amounts, serine and alanine) (Pitts and MacLeod, 1972). Serine is extracted by the liver and other tissues including skeletal muscle (Felig, 1975). Exercise does not appear to affect serine concentration by more than five percent from the mean (Felig and Wahren, 1971). Glycine is the major precursor of serine, but glycine uptake is not adequate to account for all serine, and so glycolytic intermediates are also used by the body (Brosnan, 2003).

Serine is extracted from the blood by the liver in two major ways. There is a first pass clearance effect in which the liver extracts orally dosed serine from the splanchnic circulation via the portal vein. In piglets fed with a mix of amino acids (Stoll et al., 1998) found that this first-pass metabolism in a fed state amounted to $58 \%$ of ingested serine. They also found that quantitatively more of the absorbed essential amino acids were catabolized than were incorporated into peptides and proteins. Hepatic extraction values for serine from arterial circulation of 24.1 and $55.0 \%$ were observed in rats receiving a $13 \%$ or $50 \%$ casein diet (Remesy et al., 1983). In roosters, a hepatic extraction value of $14 \%$ for serine was observed (Song et al., 2001).

In humans after $10-12 \mathrm{~h}$ fasting, It has been reported that the small extraction of serine, that is the difference of 2.4 (5.1) umol/ liter difference between portal vein and arterial concentration, was statistically insignificant (Felig and Wahren, 1971). In their study, the reported arterial serine concentration was 110.011 ) umol/liter.

The mode of liver serine uptake is extraction of serine from the hepatic artery. Presumably this occurs in both fed and fasting states. In humans, it has been showed (graphically) a drop between arterial and hepatic vein concentrations of about $20 \mathrm{umol} / \mathrm{liter}$, from a base of approximately $111 \mathrm{umol} / \mathrm{liter}$ in resting, postabsorptive (12-14 h fast) state (Felig and Wahren, 
TABLE 1 | Selected serine parameters.

\begin{tabular}{|c|c|c|c|}
\hline Measurement & Source & Value (SEM) & Species \\
\hline Volume & Pitts and Macleod. (1972) & $10,12.9 \mathrm{~L} / \mathrm{kg}$ & Dog \\
\hline \multirow[t]{6}{*}{ Baseline concentration in blood } & Stein and Moore. (1954) & 106 (4.3) umol/L & Healthy human \\
\hline & Kurt Schreier and Plückthun. (1950) & $114 \mathrm{umol} / \mathrm{L}$ & Healthy human \\
\hline & Frame. (1958) & 92.3 (6.3) umol/L & \\
\hline & Neis et al. (2017) & $97.2(4.7) \mathrm{umol} / \mathrm{L}$ & Human (radial artery) \\
\hline & Neis et al. (2017) & 130 (8.4) umol/L & Human (renal vein) \\
\hline & Wilcox et al. (1985) & 130.7 (18.5) ug/mL ${ }^{a}$ & Human \\
\hline Half-life & Wilcox et al. (1985) & $3.23(0.08) \mathrm{hrs}$ & Human \\
\hline \multirow[t]{2}{*}{ Production } & Estimated from neis data & 252 mg/h & Human \\
\hline & Kalhan and Hanson. (2012) & $1,100 \mathrm{mg} / \mathrm{h}$ & Human \\
\hline
\end{tabular}

aUnits appear to be incorrect in original.

1971). Again, using a standard value for hepatic perfusion of $1.4 \mathrm{~L} / \mathrm{min}$ (Calzia et al., 2005), this would amount to $28 \mathrm{umol} /$ minute, or $3 \mathrm{mg} / \mathrm{min}$, or $180 \mathrm{mg} / \mathrm{h}$.

There is also extraction by muscle tissue. It has been reported that the arterio-femoral vein difference of serine for subjects in a postabsorptive state (10-14 h fasting) to be $10.5 \mathrm{umol} /$ liter with a standard error of about $4 \mathrm{umol} / \mathrm{liter}$, with the base arterial level to be $110.0 \pm 11 \mathrm{umol} /$ liter (Felig and Wahren, 1971). This work also gave an estimate of total blood flow to the legs, which was 0.9 (SEM 0.05) L/min. However, if a standard value for specific blood flow to muscle of $0.08 \mathrm{~L} / \mathrm{kg} / \mathrm{min}$ and the body is assumed to be $42 \%$ muscle, a $70 \mathrm{~kg}$ human will have muscle perfusion totaling $2.4 \mathrm{~L} / \mathrm{min}$ (Calzia et al., 2005). If the serine extracted from all muscle averages the same $10.5 \mathrm{umol} / \mathrm{liter}$, this gives an approximation of $25 \mathrm{umol} / \mathrm{minute}$, or $2.6 \mathrm{mg} / \mathrm{min}$, or $156 \mathrm{mg} / \mathrm{h}$.

The fasting values of $4 \mathrm{mg} / \mathrm{min}$ released by the kidney, and 3 and $2.6 \mathrm{mg} / \mathrm{min}$ uptake into the liver and muscle, respectively, are approximations but the values were used to check our model for gross error.

TOXICOLOGY Garlick (Garlick, 2001) assessed hazards of increased oral amino acid intake and noted that data were sparse. Besides the paper by (Wilcox et al., 1985), which evaluated large doses (nearly $30 \mathrm{~g}$ equivalent in a $70 \mathrm{~kg}$ subject) in 10 control and 12 psychotic subjects and noted no adverse effects, a study of four actively psychotic schizophrenics by (Pepplinkhuizen et al., 1980) found that administering $2 \mathrm{mmol} / \mathrm{kg}$ (which would be about $13 \mathrm{~g}$ total, for a $60 \mathrm{~kg}$ subject) of serine to four female patients led to depersonalization in all, dysperception in 3, and euphoria and hallucination in one. Additional effects were noted. In all, the effects occurred about $5 \mathrm{~h}$ after administration, and lasted 3-6 h. None of the controls reacted to serine. This has implications when administering serine to trial subjects or patients with high baseline serine levels. It should be noted that serine does racemize to its enantiomer D-serine, and that D-serine is being investigated in schizophrenia - as a potential therapy (Kantrowitz et al., 2010, 2015), or as a therapy in combination with other therapies (Tsai et al., 1998) (Heresco-Levy et al., 2005).

Doses of $0.5,2.5,7.5$, and $15 \mathrm{~g}$ of serine were administered twice a day in a trial with ALS (Amytrophic Lateral Sclerosis) patients (Levine et al., 2017). While three of the 19 subjects died during the trial, this was in line with expected mortality for the cohort of ALS patients tested. Adverse effects which may be due to serine included one withdrawal due to bloating in one $15 \mathrm{~g} \mathrm{~b}$. i.d. patient.

In sum, serine is a dietary component and even at fairly elevated dosing ( $30 \mathrm{~g} /$ day $)$ showed rew reported adverse effects and those reported were minor.

The advantage of making our model "physiological," in which parameters such as serine biosynthesis rate correspond to a physical reality, allowed us to check values against reported values, and to constrain other (unreported) values.

\section{RESULTS-CLINICAL TRIAL}

The goal of the trial was to support optimal dosing for subsequent trials and to support better insight from our other modeling approaches, (e.g. GEM models of NAFLD and NASH, CITE). There were previous trials with some results, and so this trial was to improve quantitative understanding (for example of endogenous serine production) previously reported.

Ten participants were recruited within the schedule constraints imposed by the next trial and the number was determined to be adequate for purpose. All subjects were made aware of the risks inherent in the trial, and all agreed to participate. The study was performed in accordance with the Declaration of Helsinki and it was approved by the Ethics Committee at the Koc University, Istanbul, Turkey. Each subject gave written informed consent before participation in the study. The clinical trial was registered at ClinicalTrials.gov (with identifier: NCT03838822). Time limitations in recruiting limited trial subjects to males (future trials will include both sexes). One subject (subject 9) is a Type 1 diabetic and the other subjects were generally healthy. One subject (subject 10) had received the cocktail during the preceding two weeks, but that subject's results were unremarkable and very close to responses of other subjects. Demographic data are summarized in Table 1. The protocol covered five days. All dosing was oral, in the morning after an overnight fast. On the first day, all subjects received $1 \mathrm{~g}$ of nicotinamide riboside. On day two, all subjects received $3 \mathrm{~g}$ of L-carnitine. On day three, all subjects received $5 \mathrm{~g}$ of $\mathrm{N}$-acetyl cysteine. On day four, all subjects received a "cocktail" comprising the same dosing of NR, L-Carnitine, and NAC, and $20 \mathrm{~g}$ of serine. On day 5, a $20 \mathrm{~g}$ dose of serine alone was 

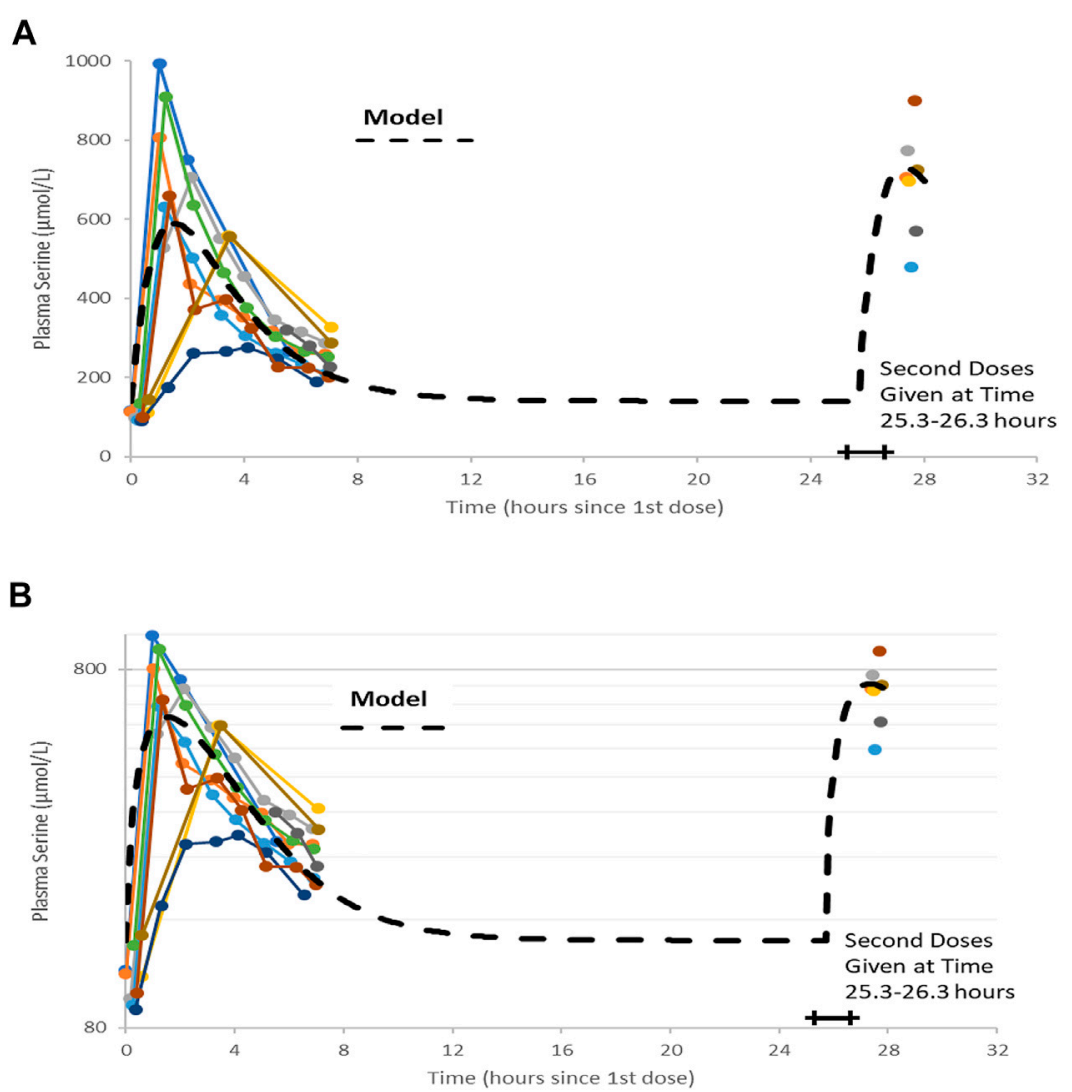

FIGURE 2 | Plots of data, time-shifted so that the initial dose time is $0 \mathrm{~h}$. This resulted in the second dose time varying between 25.3 and $26.3 \mathrm{~h}$, shown as a horizontal bar in figures. A naïve pooled model (dashed black line) is plotted with the individual data (colored dots and solid lines). (A) Linear plot. (B) Semi-logarithmic plot.

administered. Blood samples were taken every day (with number of samples per day being 2, 2, 1, 8, and 1 for days 1-5, respectively) to test the potential acute toxicity on human health and evaluate the effect of different components in the original clinical study (reported elsewhere (Zhang et al., 2020), and only relevant samples were analyzed for serine in this study. Note that the model parameter estimation included one serine data point on day 5 to evaluate the clearance after $24 \mathrm{~h}$ of cocktail supplementation.

Serine was supplied as a powder. $20 \mathrm{~g}$ doses were prepared by weight and administered after mixing with $200 \mathrm{gr}$ of water. There were no adverse effects reported by the participants during the trial. A trellis plot of the data for serine concentration over time is shown Figure 2. It is clear that a non-zero baseline value for serine is observed in all subjects. The average of the (pre-dose or baseline) serine values was 117 (6.7) umol/L, consistent with reported values given in the literature cited.

\section{MODELING AND ANALYSIS}

Figures 2A,B show the data on one plot, time-shifted so that the first dose was at time zero. The model trace shown in the figures is from a linear model, with mass action absorption of serine from a gut compartment to a plasma compartment subject to a bioavailability, mass action clearance from the plasma, and a constant endogenous biosynthesis being added to the plasma compartment. This is represented graphically in Figure 3. The modeling equations used were as follows. First, the amount of serine in the gut dose compartment, $\mathrm{D}$, is.

$$
\frac{d D}{d t}=-k_{a b s} D+\text { DoseAmt } * \delta\left(t-t_{\text {Dose }}\right) C
$$

Where $\mathrm{d}$ is the Dirac delta function and DoseAmt is the bolus dose quantity.

The equation for Serine concentration, $\mathrm{C}$, with five adjustable parameters $\left(\mathrm{V}_{\mathrm{D}}, \mathrm{F}, \mathrm{k}_{\mathrm{abs}}, \mathrm{k}_{\text {gen }}\right.$ and $\left.\mathrm{CL}\right)$ is

$$
V_{D} \frac{d C}{d t}=F k_{a b s} D+k_{g e n}-C L \cdot C
$$

Where $C$ denotes serine concentration, $V_{D}$ is the apparent volume of distribution of serine, $\mathrm{F}$ is bioavailability, $\mathrm{k}_{\mathrm{abs}}$ is a rate constant for absorption, $\mathrm{k}_{\mathrm{gen}}$ denotes a constant endogenous serine production rate, CL is serine clearance.

One modeling assumption that should be highlighted is that endogenous serine production is assumed constant. One conclusion is that the administered dose of serine is virtually cleared within $12 \mathrm{~h}$.

Note that without a pre-existing value for the volume of distribution or bioavailability, this form of equation is usually poorly conditioned and any set of parameters $\left(\mathrm{V}_{\mathrm{D}}, \mathrm{F}, \mathrm{k}_{\mathrm{abs}}, \mathrm{k}_{\mathrm{gen}}\right.$, 


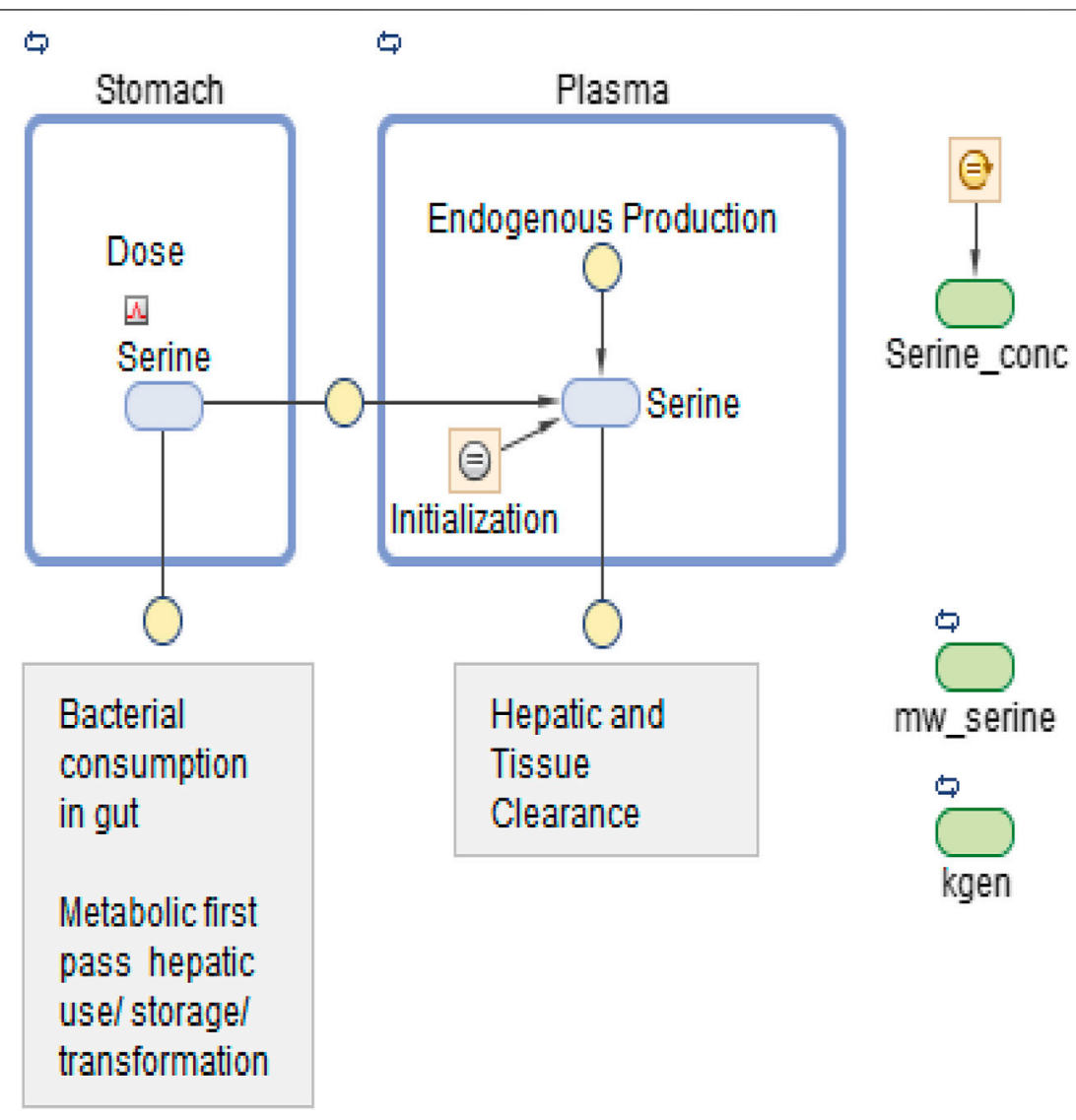

FIGURE 3 | Graphical model representation from SimBiology(R).

TABLE 2 | Trial subject demographic data.

\begin{tabular}{lccc}
$\mathbf{N}=\mathbf{1 0}$ & Weight $\mathbf{( k g )}$ & Height $\mathbf{( c m )}$ & $\mathbf{B m i}$ \\
\hline Mean & 82.8 & 177.0 & 26.4 \\
Std dev & 16.5 & 8.3 & 4.5 \\
Median & 78.9 & 175.0 & 25.3 \\
Minimum & 61.0 & 166.0 & 19.4 \\
Maximum & 114.8 & 192.5 & 34.5
\end{tabular}

and CL) that fits data could be scaled by an arbitrary amount to fit the data equally well. Nonetheless, a least-squares fitting process using naïve pooled technique did converge to a volume of about $1 \mathrm{~L}$. We know that this is likely not correct, as (Pitts and MacLeod, 1972) estimated volumes for dogs of approximately $160 \mathrm{~L}$. Parameter values are shown in Table 2. The individual data, and the naive pooled model results are plotted in Figures 2A,B.

The terminal clearance coefficient $\left(C L / V_{D}\right.$, i.e., the rate of endogenous generation) is $0.48 / \mathrm{hr}$. This is equivalent to a half-life of $1.45 \mathrm{~h}$, a bit lower than reported by (Wilcox et al., 1985). The rate of endogenous generation is calculated ( $\mathrm{CL} *$ baseline) as $6.6 \mathrm{mg} / \mathrm{h}$, significantly lower than discussed above. This assumes a volume of distribution of about $1 \mathrm{~L}$, though, which is significantly at odds with the 199 and $260 \mathrm{~L}$ values for dogs cited by (Pitts and MacLeod, 1972).

In addition to the non-physiological value for volume, the previous fit uses a naïve pooled-date approach and hence does not represent any subject very well, as the averaging process distorts absorption and clearance parameters and yields unrepresentative values for, for example, predicted maximum concentration, or Cmax. This motivated the use of population modeling.

The model may be modified to improve conditioning and to allow more detailed representation of the physiology discussed above so as to allow the use of a wider range of data. Also, one desired use for the model was to simulate clinical trials. Models that represent an average patients, but not any individual patient are not adequate for these tasks.

Model fitting without prior knowledge of volume (or bioavailability) will require modification to equation 2 to improve conditioning as shown:

$$
\frac{d C}{d t}=\frac{F}{V_{D}} k_{a b s} D+\frac{k_{\text {gen }}}{V_{D}}-\frac{C L}{V_{D}} C
$$

This reduces the degrees of freedom by one, as the fitting must solve only for four adjustable constant parameters $\left(F / V_{D}, k_{a b s}\right.$, $\mathrm{k}_{\mathrm{gen}} / \mathrm{V}_{\mathrm{D}}$ and $\mathrm{CL} / \mathrm{V}_{\mathrm{D}}$ ). 


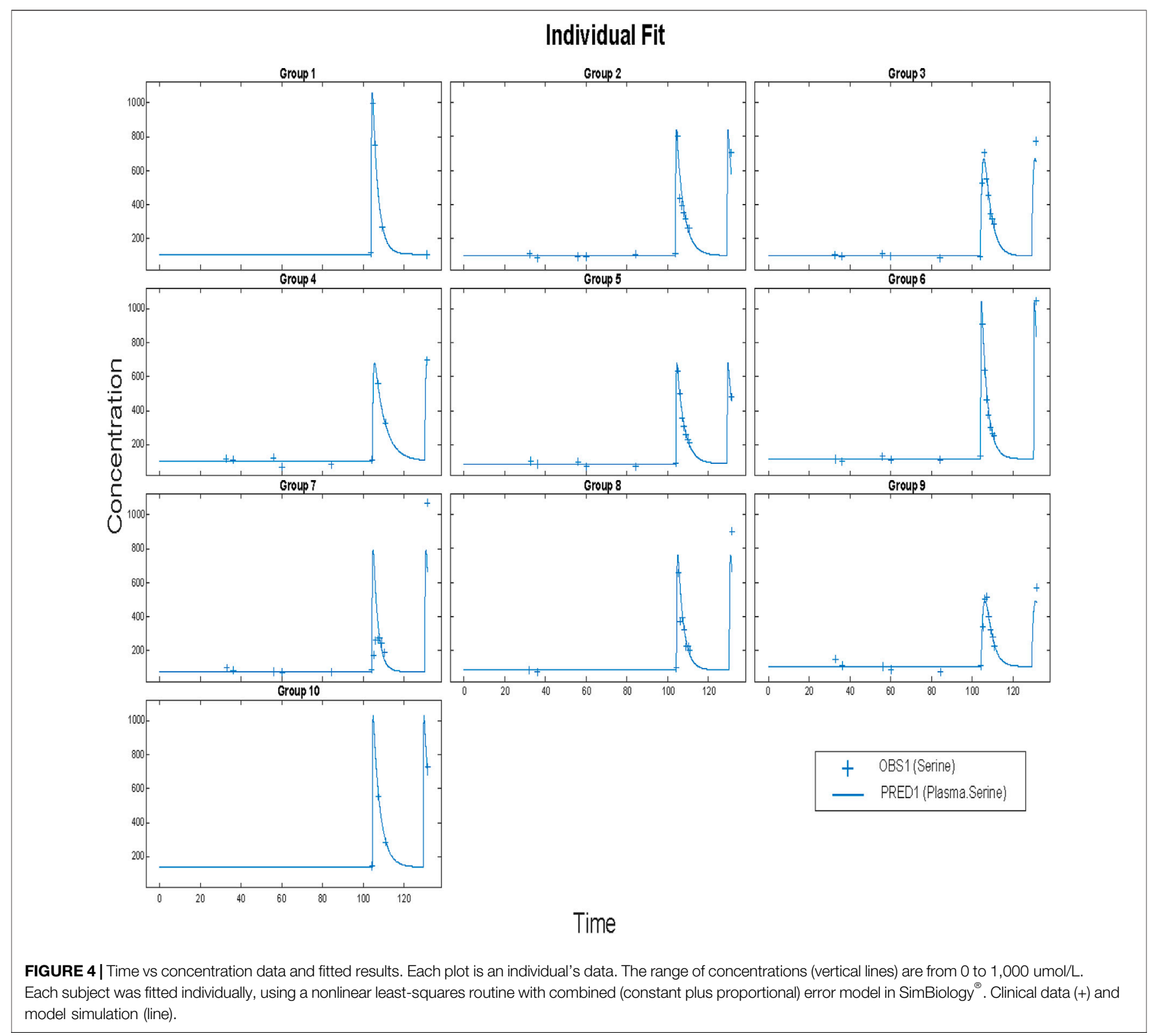

TABLE 3 | Naive pooled fitted parameters for the poorly conditioned model shown in Figures 2A,B.

\begin{tabular}{|c|c|c|c|c|c|}
\hline Dose & $\mathbf{K a}$ & $\mathbf{F}$ & CL & Baseline & $\mathbf{v}$ \\
\hline- & Absorption & Bioavailability & Clearance & Concentration & Volume \\
\hline 20 & 0.829 & 0.00452 & 0.446 & 1.474 & 0.936 \\
\hline
\end{tabular}

To generate parameter estimates and distributions, and to understand parametric distribution and confidence intervals for model prediction, we proceeded to use population pharmacometric approaches to modeling (Mold and Upton, 2013; Owen and Fiedler-Kelly, 2014; Upton and Mold, 2014, 2014).
The model was first implemented in SimBiology (MathWorks Inc., Natick MA, United States). SimBiology allows a graphical approach to model building. The picture of the model has underlying mathematics, and fitting and data manipulation can be done using integrated software. The model is pictured in Figure 3. 
TABLE 4 | Parameters from individual fit.

\begin{tabular}{lcccc}
\hline & Kabs & $\mathbf{F} / \mathbf{V}_{\mathbf{D}} \times \mathbf{1}, \mathbf{0 0 0}$ & $\mathbf{C L} / \mathbf{V}_{\mathbf{D}}$ & $\mathbf{k}_{\text {gen }} / \mathbf{V}_{\mathbf{D}}$ \\
\hline Mean & 4.67 & 4.94 & 0.346 & 35.8 \\
Std dev & 3.33 & 0.96 & 0.087 & 11.0 \\
Minimum & 0.51 & 3.47 & 0.198 & 20.6 \\
Maximum & 15.6 & 6.51 & 0.505 & 54.5 \\
Units & 1/hour & 1/Liter & 1/hour & Umol/(hr*liter)
\end{tabular}

Before applying population methods, a first step in fitting this model was to fit each subject individually. This was done using the nonlinear least-squares (lsqnonlin) routine. The mixed (constant plus proportional) error model was found to give the best results. The results are shown in Figure 4 and parameter estimates are given in Table 3.

Again, the mean values given cannot be guaranteed to actually represent any individual. That is, using these parameters in a simulation may result in an outcome that is not physiologically feasible or reasonable. Furthermore, the method used gave a physiologically unreasonable volume of distribution, due to the absence of iv data for serine administration. We could find no such data. So in subsequent fits we estimated ratios $(\mathrm{CL} / \mathrm{V}$, for example). A way to achieve mean parameters that are representative is to use a population approach. The nlmefit (non-linear mixed effect modeling) routine in SimBiology (MathWorks Inc., Natick, MA) was used using a combined (constant plus proportional) error model. The approach yielded the fits shown below:

The resulting parameters are given in Table 4. What is striking is just how small the variabilities for several parameters are. Most of the variability is in the bioavailability $(\mathrm{F} / \mathrm{V})$ parameter. The parameters were estimated as following a lognormal distribution, and so the parameter values estimated as the natural logarithm of the parameter, with standard errors are in logarithmic terms. To give ranges in meaningful units, the exponential of the nominal value and the nominal value plus and minus one standard error were calculated. Model results are plotted in Figure 5.

To check these results, the same model was programmed in NONMEM $^{\circledR}$ (ICON Plc, Ellicott City, MD). Parameter distribution was implemented as lognormal. The proportional error model gave nearly the same results as the mixed model, and so proportional error was used. The results for the proportional error model are given in Table 5.

The Eta values that are shown as small were so small as to not allow noticeable variability (no change in three significant figures) and could be fixed in the estimation scheme (Figure 6; Table 6). We note that the variability in the NONMEM parameters yields

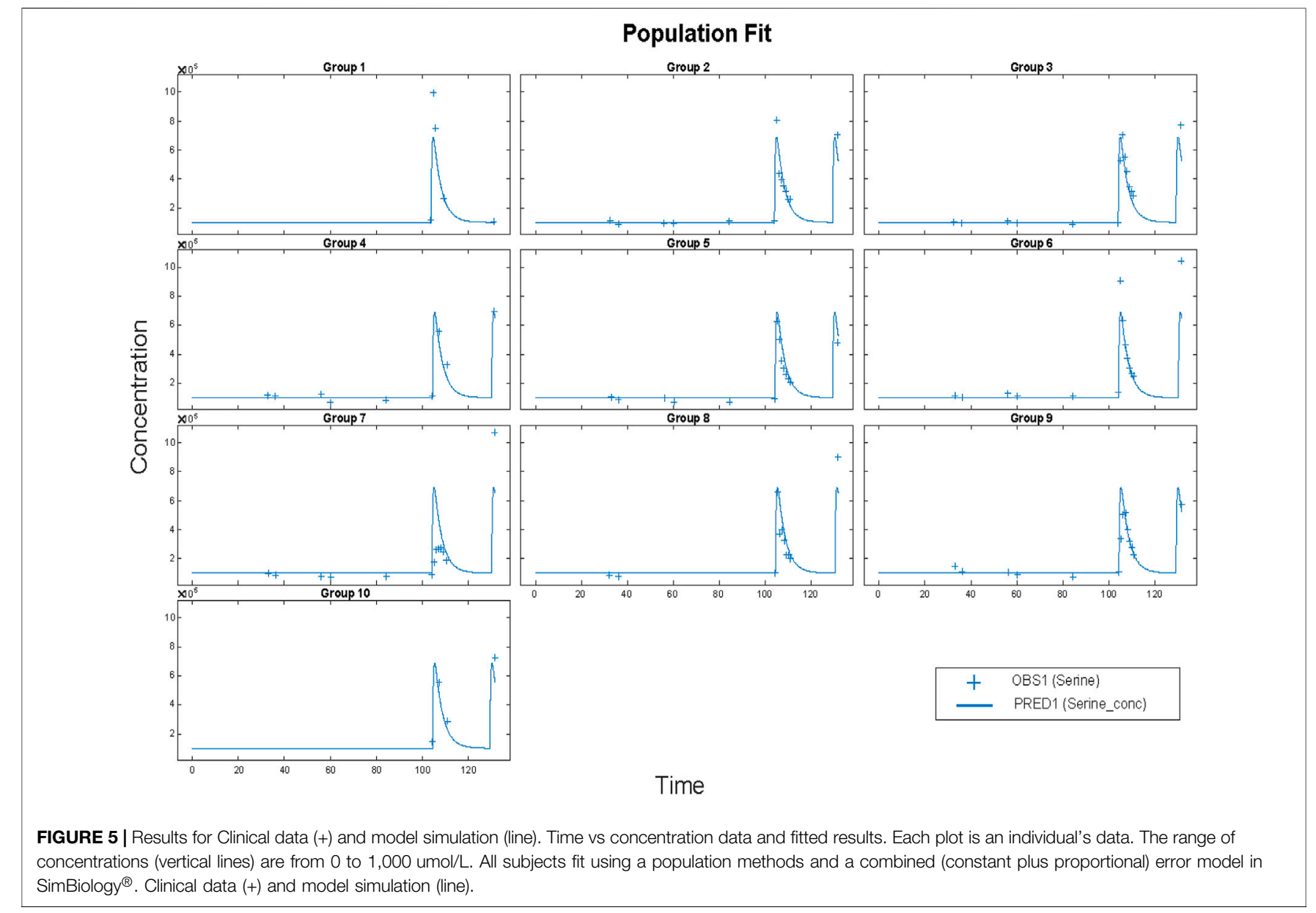


TABLE 5 | Parameters from population fit using SimBiology ${ }^{\circledR}$ nlmefit with proportional error model.

\begin{tabular}{|c|c|c|c|c|}
\hline & Kabs & $F / V_{D} \times 1,000$ & $C L / V_{D}$ & $\mathbf{k}_{\text {gen }} / \mathbf{V}_{\mathrm{D}}$ \\
\hline Population & 2.88 & 4.29 & 0.288 & 29.6 \\
\hline Pop-SEM & 1.88 & 3.96 & 0.266 & 27.0 \\
\hline Pop +SEM & 4.42 & 4.64 & 0.312 & 32.3 \\
\hline Minimum & 2.88 & 3.47 & 0.288 & 29.6 \\
\hline Maximum & 2.89 & 4.87 & 0.288 & 29.6 \\
\hline Units & 1/hour & 1/Liter & 1/hour & Umol/(hr*liter) \\
\hline
\end{tabular}

qualitatively different result than observed with nlmefit. One plausible explanation is that the two approaches found different local minima (Ricardo Paxson and Florian Augustin of MathWorks Inc., personal communication) as parameter variance estimates in nlmefit initially are set to zero, while in $\mathrm{NONMEM}^{\circledR}$, these values may be initialized via the OMEGA vector or matrix.

We evaluated whether a second compartment improved the model. The improvement in the log-likelihood-based NONMEM metric ( 967.5 vs 979.785 for the one compartment model) lead to a difference of 12.2 which is more than the threshold of 5.99 (Fisher and Shafer, 2007) and would justify the addition of two additional parameters (rate-law constants k23 and k32). However, the fitted value of these parameters were four orders of magnitude less than clearance and absorption rate constants and resulted in other parameter estimates changing only in the third decimal place with a change in predicted values that was trivial in relation to serine concentration. The additional compartment complexity was rejected on this basis.
We evaluated weight and height as potential covariates. Of the two parameters that showed variation between subjects, neither was shown to be correlated in any way to either metric. Likewise, there were no meaningful trends observed between other metrics (e.g. residual values) and weight and height. Our final model did not include covariates.

\section{DISCUSSION}

By using a model that was consistent with physiology, a broad range of published data for serine was able to be exploited. Model structure was improved, and we were able to build confidence in some fitted parameters (such as endogenous production rate). Animal extraction data (Neis et al., 2017) were used to estimate bioavailability ( $60 \%)$ and ultimate hepatic serine uptake fraction (75\%). We were able to reject reported volume of distribution values from animal data (Pitts and MacLeod, 1972) as inappropriate for scaling to humans. Without intravenous data, a value for volume of distribution was estimated for serine $(\sim 140 \mathrm{~L})$.

The dosing of $20 \mathrm{~g}$ QD in normal patients did not elicit any adverse effects in our clinical trial. The work of Wilcox et al. (Wilcox et al., 1985) noted no adverse effects in normal and psychotic subjects for a dose of $4 \mathrm{mM} / \mathrm{kg}$, which is equivalent to $0.424 \mathrm{~g} / \mathrm{kg}$, or a $29.7 \mathrm{~g}$ dose for a $70 \mathrm{~kg}$ subject. Wilcox et al. (Wilcox et al., 1985) did note a higher baseline serine value, and lower SHMT activity, in psychotic subjects, and (Pepplinkhuizen et al., 1980) did observe schizophrenia-like psychoses induced in
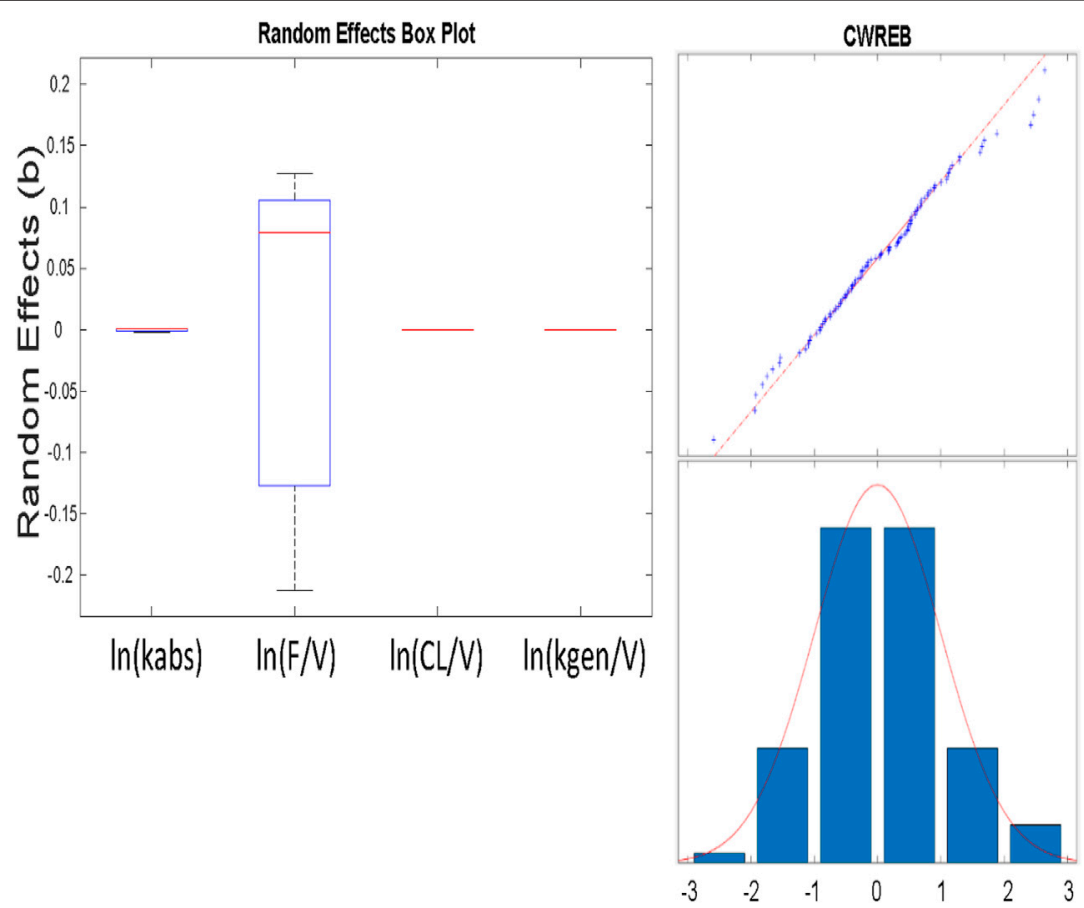

FIGURE 6 |A) (above) Box and whisker plot of estimated parameters from population fit. Most variability is in the FND parameter. B) (at right) Plot of residuals to evaluate normality using a combined (constant plus proportional) error model. The combined model gave improved normality in the "tails" at both low and high values shown here, as opposed to a constant error model. 
TABLE 6 | Population fit parameters from NONMEM.

\begin{tabular}{lcccc}
\hline & Kabs & $\mathbf{F} / \mathbf{V}_{\mathbf{D}} \times \mathbf{1}, \mathbf{0 0 0}$ & $\mathbf{C L} / \mathbf{V}_{\mathbf{D}}$ & $\mathbf{k}_{\mathbf{g e n}} / \mathbf{V}_{\mathbf{D}}$ \\
\hline Population & 2.75 & 4.58 & 0.312 & 32.1 \\
Eta value & 0.921 & Small (FIXed) & 0.0135 & Small (FIXed) \\
Units & 1/hour & 1/Liter & $1 /$ hour & Umol/(hr*liter)
\end{tabular}

four active female schizophrenics $(2 \mathrm{mM} / \mathrm{kg}$, or $0.212 \mathrm{~g} / \mathrm{kg}$, equivalent to $12.7 \mathrm{~g}$ dose in a $60 \mathrm{~kg}$ women). This suggests, but does not prove, that a dose of $20 \mathrm{~g}$ or less may be tolerated in those who are not actively psychotic. Given that biopsy and/or tracer studies for SHMT activity are intrusive, a baseline serine value should be considered as an inclusion/ exclusion test for future trials.

With that said, the $75 \%$ overall hepatic extraction with a $20 \mathrm{~g}$ dose is equivalent to $15 \mathrm{~g}$ absorbed by the liver. Averaging this over $24 \mathrm{~h}$ yields $10.4 \mathrm{mg} / \mathrm{min}$ - a bit more than twice the current value of endogenous production, from clinical measurements. Thus, a $20 \mathrm{~g}$ daily dose is expected to approximately triple hepatic serine uptake. Alternately, an approximately $12 \mathrm{~g}$ dose twice a day could be considered, due to the quick clearance of serine.

Systems modeling allowed the use of a broader set of data to expand our understanding of trial results. Reported parameters were used to rule out our first naïve pooled model, and to bound parameters in our population PK model. The population PK model was then used to inform our dosing decision for an upcoming trial. The modeling also highlighted the need to determine volume of distribution and bioavailability.

What was not considered was the variation of serine release and uptake during the day. We believe that this variability is inconsequential for our purpose of dose-setting, but the model could be augmented should this variation be relevant. We did not include the extraction of serine by the brain, but this could be easily analyzed using a modification of the current model and relevant trial data, for example using the results of (Levine et al., 2017), and a completed trial of serine in neuropath (Fridman et al., 2019).

A key interest in dose determination for serine is estimating the amount absorbed by the liver. Hepatic extraction will be both first-pass, from splanchnic circulation into the hepatic portal vein, and also system extraction from arterial circulation. Supposing that the 24.1 or 55 percent extraction range observed by (Remesy et al., 1983) in rats applies to humans, and assuming other use (for example, bacterial consumption in the gut) is negligible, this would imply a standard oral bioavailability values of 75.9 and $45 \%$. Using the fitted value for F/V of 0.00429 gives values of volume of distribution of 177 and $104 \mathrm{~L}$. The question is whether these numbers and ranges are physiological.

It has been reported that the values for volume of distribution of 199 and $360 \mathrm{~L}$ for dogs weighing 20 and $28 \mathrm{~kg}(10 \mathrm{~L} / \mathrm{kg}$ and $12.9 \mathrm{~L} / \mathrm{kg}$, respectively, must be scaled (Pitts and MacLeod, 1972). One source gives blood volume in dogs as $79 \mathrm{ml}$ of blood per $\mathrm{kg}$ of body weight (Courtice, 1943). From this, we can calculate blood volume estimates of 1.580 and $2.212 \mathrm{~L}$. Allometric scaling principles suggest that these values might be scaled proportionally to body weight (Holford and Anderson, 2007), yielding (for both dogs) a value of about $5.5 \mathrm{~L}$ of blood for a $70 \mathrm{~kg}$ human, only about $10 \%$ higher than the commonly used value of $5 \mathrm{~L}$. The volume of distributions from dogs can be scaled proportionally to weight, yielding 700 and $900 \mathrm{~L}$. Using the average value of $800 \mathrm{~L}$, and 0.00429 for $\mathrm{F} / \mathrm{V}$ gives an infeasible bioavailability of $340 \%$. This suggests that the volume of distribution of serine reported for dogs (Pitts and MacLeod, 1972) is not appropriate or useful for humans, or at least that allometric scaling was not correct. Because of this, and because we suspect that extraction may be a more intrinsically conserved between species, the hepatic extraction values reported in rats (Remesy et al., 1983) were used to generate bioavailability. A bioavailability of about 0.6 (roughly the average between the 0.759 and 0.45 values in rats) yielded an effective volume of distribution of about $140 \mathrm{~L}$.

Of key interest is the fate of the approximately $60 \%$ of serine that is not initially absorbed by the liver. Recall the estimate above that the liver consumed about $3 \mathrm{mg} / \mathrm{min}$, and the muscle tissue about $2.6 \mathrm{mg} / \mathrm{min}$. Assuming that these are the major consumers of arterial serine yields an estimate of arterial serine clearance of $46 \%$ by muscle and $54 \%$ by the liver. These values suggest that the liver extracts about $73 \%$ ( $40 \%$ initially, and another $46 \%$ of the $60 \%$ of serine that ends up in plasma) of ingested serine, with the rest going to muscle. This number is probably high, as some of the arterial serine is likely extracted by tissues other than muscle.

The population fit of endogenous serine generation was estimated to be $29.6 \mathrm{umol} / \mathrm{L} / \mathrm{hr}$, as $\mathrm{kgen} / \mathrm{V}$. When multiplied by the estimated volume of $140 \mathrm{~L}$, this gives $440.0 \mathrm{mg} / \mathrm{h}$. This must be compared with the $252 \mathrm{mg} / \mathrm{h}$ estimated from (Neis et al., 2017), and the $1,110 \mathrm{mg} / \mathrm{h}$ estimated from the work of (Kalhan and Hanson, 2012) which was felt to be high. Given differences in protocols, subjects, and variability of measurements, these value does not compare unfavorably, and provide some confidence in the model analysis.

If $440 \mathrm{mg}$ is generated endogenously every hour, and the liver accounts for the uptake of $54 \%$ of that amount, normal liver uptake of serine is about $6 \mathrm{~g}$ of serine per day. An oral dose of $8 \mathrm{~g} /$ day $(6 \mathrm{~g} / 73 \%$ liver utilization for oral dosing) could approximately double liver uptake, and a dose of $16 \mathrm{~g}$ /day could be expected to triple liver uptake, provided the extraction values considered here did not change. Earlier experiments with once-per-day $20 \mathrm{~g}$ dosing (Mardinoglu et al., 2017) gave good results in some subjects, weaker responses in others. Noting that daily dosing up to about $30 \mathrm{~g}$ have been administered to normal subjects without adverse effects, and desiring a stronger effect suggested that a prudent upper bound would be a total of $25 \mathrm{~g}$ per day. Given that the serine is apparently cleared quickly and a sustained metabolic effect is desired, the recommended $25 \mathrm{~g}$ dose could be administered as $12.5 \mathrm{~g}$, twice a day. This was the recommendation for the upcoming trial. A more comprehensive clinical trial is planned and will be executed. A portion of that trial will include intravenous dosing to allow a better determination of bioavailability. In addition, we expect to use tracer studies to confirm hepatic extraction values derived from the model.

We have fit new serine data to a model representing physiology (serine synthesis and clearance). The parameters of 
the model correspond to physiological quantities, allowing comparisons and building confidence in the model, the methods used, and the data reported. The generally good fit of the model built confidence in structure and fitted parameters, while the agreement between the model parameters and reported values of physiological constants gave additional model validation. The dose determined using the model was selected for future trials.

\section{DATA AVAILABILITY STATEMENT}

The original contributions presented in the study are included in the article/Supplementary Material, further inquiries can be directed to the corresponding authors.

\section{ETHICS STATEMENT}

The studies involving human participants were reviewed and approved by the Ethics Committee of Koc University, Istanbul,

\section{REFERENCES}

Anderson, B. J., and Holford, N. H. (2007). Mechanism-Based Concepts of Size and Maturity in Pharmacokinetics. Annu. Rev. Pharmacol. Toxicol. 48, 303-332. doi:10.1146/annurev.pharmtox.48.113006.094708

Brosnan, J. T. (2003). Interorgan Amino Acid Transport and its Regulation. J. Nutr. 133, 2068S-2072S. doi:10.1093/jn/133.6.2068s

Calzia, E., Iványi, Z., and Radermacher, P. (2005). "Determinants of Blood Flow and Organ Perfusion," in Functional Hemodynamic Monitoring (Springer), 19-32.

Courtice, F. C. (1943). The Blood Volume of Normal Animals. J. Physiol. 102, 290-305. doi:10.1113/jphysiol.1943.sp004035

de Koning, T. J., Snell, K., Duran, M., Berger, R., Poll-The, B.-T., and Surtees, R. (2003). L-serine in Disease and Development. Biochem. J. 371, 653-661. doi:10. 1042/bj20021785

Felig, P. (1975). Amino Acid Metabolism in Man. Annu. Rev. Biochem. 44, 933-955. doi:10.1146/annurev.bi.44.070175.004441

Felig, P., and Wahren, J. (1971). Amino Acid Metabolism in Exercising Man. J. Clin. Invest. 50, 2703-2714. doi:10.1172/jci106771

Fisher, Dennis., and Shafer, Steven. (2007). Fisher/Shafer NONMEM Workshop Pharmacokinetic and Pharmacodynamic Analysis with NONMEM Basic Concepts. Ghent, Belgium: Het Pand.

Frame, E. G. (1958). The Levels of Individual Free Amino Acids in the Plasma of Normal Man at Various Intervals after a High-Protein Meal1. J. Clin. Invest. 37, 1710-1723. doi:10.1172/jci103763

Fridman, V., Suriyanarayanan, S., Novak, P., David, W., Macklin, E. A., McKennaYasek, D., et al. (2019). Randomized Trial of L-Serine in Patients with Hereditary Sensory and Autonomic Neuropathy Type 1. Neurology 92, e359-e370. doi:10.1212/wnl.0000000000006811

Gantner, M. L., Eade, K., Wallace, M., Handzlik, M. K., Fallon, R., Trombley, J., et al. (2019). Serine and Lipid Metabolism in Macular Disease and Peripheral Neuropathy. N. Engl. J. Med. 381, 1422-1433. doi:10.1056/nejmoa1815111

Garlick, P. J. (2001). Assessment of the Safety of Glutamine and Other Amino Acids. J. Nutr. 131, 2556S-2561S. doi:10.1093/jn/131.9.2556s

Garofalo, K., Penno, A., Schmidt, B. P., Lee, H.-J., Frosch, M. P., von Eckardstein, A., et al. (2011). Oral L-Serine Supplementation Reduces Production of Neurotoxic Deoxysphingolipids in Mice and Humans with Hereditary Sensory Autonomic Neuropathy Type 1. J. Clin. Invest. 121, 4735-4745. doi:10.1172/jci57549

Heresco-Levy, U., Javitt, D. C., Ebstein, R., Vass, A., Lichtenberg, P., Bar, G., et al. (2005). D-serine Efficacy as Add-On Pharmacotherapy to Risperidone and
Turkey. The patients/participants provided their written informed consent to participate in this study.

\section{AUTHOR CONTRIBUTIONS}

$A M$ and JRB conducted the study. JRB and EB developed the method. JRB and EB performed most of the analysis and CZ helped in the analysis. $\mathrm{HT}$, JN, JB, and MU provided resources for the experiment of this study. JRB wrote the paper and $\mathrm{EB}$, $\mathrm{CZ}, \mathrm{HT}, \mathrm{JN}, \mathrm{JB}, \mathrm{MU}$, and $\mathrm{AM}$ were involved in editing the paper.

\section{ACKNOWLEDGMENTS}

The authors are grateful to Mr. Ricardo Paxson and Dr Florian Augustin of Mathworks, Inc. for their helpful criticisms and insight into the Simbiology nlme fitting routine.

Olanzapine for Treatment-Refractory Schizophrenia. Biol. Psychiatry 57, 577-585. doi:10.1016/j.biopsych.2004.12.037

Kalhan, S. C., and Hanson, R. W. (2012). Resurgence of Serine: an Often Neglected but Indispensable Amino Acid. J. Biol. Chem. 287, 19786-19791. doi:10.1074/ jbc.r112.357194

Kantrowitz, J. T., Malhotra, A. K., Cornblatt, B., Silipo, G., Balla, A., Suckow, R. F. et al. (2010). High Dose D-Serine in the Treatment of Schizophrenia. Schizophrenia Res. 121, 125-130. doi:10.1016/j.schres.2010.05.012

Kantrowitz, J. T., Woods, S. W., Petkova, E., Cornblatt, B., Corcoran, C. M., Chen, H., et al. (2015). D-serine for the Treatment of Negative Symptoms in Individuals at Clinical High Risk of Schizophrenia: a Pilot, Double-Blind, Placebo-Controlled, Randomised Parallel Group Mechanistic Proof-OfConcept Trial. The Lancet Psychiatry 2, 403-412. doi:10.1016/s22150366(15)00098-x

Levine, T. D., Miller, R. G., Bradley, W. G., Moore, D. H., Saperstein, D. S., Flynn, L. E., et al. (2017). Phase I Clinical Trial of Safety of L-Serine for ALS Patients. Amyotroph. Lateral Scler. Frontotemporal Degeneration 18, 107-111. doi:10. 1080/21678421.2016.1221971

Mardinoglu, A., Agren, R., Kampf, C., Asplund, A., Uhlen, M., and Nielsen, J. (2014). Genome-scale Metabolic Modelling of Hepatocytes Reveals Serine Deficiency in Patients with Non-alcoholic Fatty Liver Disease. Nat. Commun. 5, 3083. doi:10.1038/ncomms4083

Mardinoglu, A., Bjornson, E., Zhang, C., Klevstig, M., Söderlund, S., Ståhlman, M., et al. (2017). Personal Model-assisted Identification of NAD + and Glutathione Metabolism as Intervention Target in NAFLD. Mol. Syst. Biol. 13, 916. doi:10. $15252 / \mathrm{msb} .20167422$

Mardinoglu, A., Ural, D., Zeybel, M., Yuksel, H. H., Uhlén, M., and Borén, J. (2019). The Potential Use of Metabolic Cofactors in Treatment of NAFLD. Nutrients 11, 1578. doi:10.3390/nu11071578

Mould, D. R., and Upton, R. N. (2013). Basic Concepts in Population Modeling, Simulation, and Model-Based Drug Development-Part 2: Introduction to Pharmacokinetic Modeling Methods. CPT Pharmacomet. Syst. Pharmacol. 2, e38. doi:10.1038/psp.2013.14

Neis, E. P. J. G., Sabrkhany, S., Hundscheid, I., Schellekens, D., Lenaerts, K., Olde Damink, S. W., et al. (2017). Human Splanchnic Amino-Acid Metabolism. Amino Acids 49, 161-172. doi:10.1007/s00726-016-2344-7

Owen, J. S., and Fiedler-Kelly, J. (2014). Introduction to Population Pharmacokinetic/Pharmacodynamic Analysis with Nonlinear Mixed Effects Models. John Wiley \& Sons. doi:10.1002/9781118784860

Pepplinkhuizen, L., Blom, W., Bruinvels, J., and Moleman, P. (1980). Schizophrenia-like Psychosis Caused by a Metabolic Disorder. The Lancet 315, 454-456. doi:10.1016/s0140-6736(80)91000-4 
Pitts, R., and MacLeod, M. (1972). Synthesis of Serine by the Dog Kidney In Vivo. Am. J. Physiology-Legacy Content 222, 394-398. doi:10.1152/ajplegacy.1972.222.2.394

Remesy, C., Fafournoux, P., and Demigne, C. (1983). Control of Hepatic Utilization of Serine, glycine and Threonine in Fed and Starved Rats. J. Nutr. 113, 28-39. doi:10.1093/jn/113.1.28

Scherer, S. S. (2011). The Debut of a Rational Treatment for an Inherited Neuropathy? J. Clin. Invest. 121, 4624-4627. doi:10.1172/jci60511

Schreier, K., and Plückthun, U. H. (1950). Untersuchungen über den Gehalt an freien Aminosäuren im Serum und Urin. Biochem. Z. 320, 447-465.

Sim, W.-C., Yin, H.-Q., Choi, H.-S., Choi, Y.-J., Kwak, H. C., Kim, S.-K., et al. (2015). L-serine Supplementation Attenuates Alcoholic Fatty Liver by Enhancing Homocysteine Metabolism in Mice and Rats. J. Nutr. 145, 260-267. doi:10.3945/jn.114.199711

Song, Z., Beers, K., Dibner, J. J., Vázquez-Añón, M., McNew, R., and Bottje, W. (2001). The Hepatic Extraction of Plasma Free Amino Acids and Response to Hepatic Portal Venous Infusion of Methionine Sources in Anesthetized SCWL Males (Gallus domesticus). Comp. Biochem. Physiol. B: Biochem. Mol. Biol. 130, 237-250. doi:10.1016/s1096-4959(01)00430-4

Stein, W. H., and Moore, S. (1954). The Free Amino Acids of Human Blood Plasma. J. Biol. Chem. 211, 915-926. doi:10.1016/s0021-9258(18)71179-4

Stoll, B., Henry, J., Reeds, P. J., Yu, H., Jahoor, F., and Burrin, D. G. (1998). Catabolism Dominates the First-Pass Intestinal Metabolism of Dietary Essential Amino Acids in Milk Protein-Fed Piglets. J. Nutr. 128, 606-614. doi:10.1093/jn/ 128.3.606

Tsai, G., Yang, P., Chung, L.-C., Lange, N., and Coyle, J. T. (1998). D-serine Added to Antipsychotics for the Treatment of Schizophrenia. Biol. Psychiatry 44, 1081-1089. doi:10.1016/s0006-3223(98)00279-0
Upton, R. N., and Mould, D. R. (2014). Basic Concepts in Population Modeling, Simulation, and Model-Based Drug Development: Part 3-Introduction to Pharmacodynamic Modeling Methods. CPT Pharmacomet. Syst. Pharmacol. 3, e88. doi:10.1038/psp.2013.71

Wilcox, J., Waziri, R., Sherman, A., and Mott, J. (1985). Metabolism of an Ingested Serine Load in Psychotic and Nonpsychotic Subjects. Biol. Psychiatry 20, 41-49. doi:10.1016/0006-3223(85)90133-7

Zhang, C., Bjornson, E., Arif, M., Tebani, A., Lovric, A., Benfeitas, R., et al. (2020). The acute effect of metabolic cofactor supplementation: a potential therapeutic strategy against non-alcoholic fatty liver disease. Mol. Syts. Biol. 16(4), e9495. doi: $10.15252 / \mathrm{msb} .209495$

Conflict of Interest: JRB is the founder of Clermont Bosley LLC. AM, JB, and MU are the founder and shareholders of ScandiBio Therapeutics and ScandiEdge Therapeutics.

The remaining authors declare that the research was conducted in the absence of any commercial or financial relationships that could be construed as a potential conflict of interest.

Copyright (c) 2021 Bosley, Björnson, Zhang, Turkez, Nielsen, Uhlen, Borén and Mardinoglu. This is an open-access article distributed under the terms of the Creative Commons Attribution License (CC BY). The use, distribution or reproduction in other forums is permitted, provided the original author(s) and the copyright owner(s) are credited and that the original publication in this journal is cited, in accordance with accepted academic practice. No use, distribution or reproduction is permitted which does not comply with these terms. 